\title{
Intact Detection of Highly Occluded Immature Tomatoes on Plants Using Deep Learning Techniques
}

\author{
Yue $\mathrm{Mu}^{1,2, *(\mathbb{D}}$, Tai-Shen Chen ${ }^{3}$, Seishi Ninomiya ${ }^{1,2}$ and Wei Guo ${ }^{2, *(\mathbb{D}}$ \\ 1 Plant Phenomics Research Center, Jiangsu Collaborative Innovation Center for Modern Crop Production, \\ Nanjing Agricultural University, No. 1 Weigang, Nanjing 210095, China; snino@g.ecc.u-tokyo.ac.jp \\ 2 International Field Phenomics Research Laboratory, Institute for Sustainable Agro-ecosystem Services, \\ The University of Tokyo, 1-1-1 Midori-cho, Nishi-Tokyo, Tokyo 188-0002, Japan \\ 3 Graduate School of Agricultural and Life Sciences, The University of Tokyo, 1-1-1 Yayoi, Bunkyo, \\ Tokyo 113-8657, Japan; chen.taishen@ut-biomet.org \\ * Correspondence: yuemu@njau.edu.cn (Y.M.); guowei@g.ecc.u-tokyo.ac.jp (W.G.); \\ Tel.: +86-25-84-399-651 (Y.M.); +81-42-4631-613 (W.G.); Fax: +86-25-84-396-434 (Y.M.); +81-42-464-4391 (W.G.)
}

Received: 19 April 2020; Accepted: 19 May 2020; Published: 25 May 2020

\begin{abstract}
Automatic detection of intact tomatoes on plants is highly expected for low-cost and optimal management in tomato farming. Mature tomato detection has been wildly studied, while immature tomato detection, especially when occluded with leaves, is difficult to perform using traditional image analysis, which is more important for long-term yield prediction. Therefore, tomato detection that can generalize well in real tomato cultivation scenes and is robust to issues such as fruit occlusion and variable lighting conditions is highly desired. In this study, we build a tomato detection model to automatically detect intact green tomatoes regardless of occlusions or fruit growth stage using deep learning approaches. The tomato detection model used faster region-based convolutional neural network (R-CNN) with Resnet-101 and transfer learned from the Common Objects in Context (COCO) dataset. The detection on test dataset achieved high average precision of $87.83 \%$ (intersection over union $\geq 0.5)$ and showed a high accuracy of tomato counting $\left(\mathrm{R}^{2}=0.87\right)$. In addition, all the detected boxes were merged into one image to compile the tomato location map and estimate their size along one row in the greenhouse. By tomato detection, counting, location and size estimation, this method shows great potential for ripeness and yield prediction.
\end{abstract}

Keywords: precision horticulture; deep learning; image analysis; robotic harvesting

\section{Introduction}

Tomatoes are the second most important horticultural crop [1] in terms of yield, with total production of more than 180 million tonnes across the world (FAO STAT 2017 [2]). The cultivation of tomatoes is one of the most profitable agricultural businesses because tomatoes are self-compatible and have a short life cycle [3]. For high yield and good quality, the crop needs precision management of water throughout the growing period [4], as well as fertilizer and pest control [5]. For example, due to the nonuniform flowering and ripening stages, precise irrigation is needed to ripen immature plants and avoid damage to mature plants [4]. In addition, depending on the ultimate use of the tomatoes, they may be harvested at different stages of ripeness. Tomatoes with "mature green" (medium green to light green) and dim pink colour are shipped, those with reddish-pink colour are sold locally, and those with dark red colour are processed [6]. Thus, optimal tomato cultivation requires tomato-on-plant detection to provide the fruit location and ripening status on spatial variation on which to base agronomic decisions [7]. To inform harvest resourcing and management, and marketing, tomato yield prediction requires dynastically and precise monitoring of tomato number, size, and ripening 
status [8-10]. The combination of computer vision and the Internet of Things (IoT) makes it possible to accurately monitor the growth of greenhouse crops [11]. In addition, as the agricultural population decreases and ages, robots are being considered as replacements for humans to undertake manual and tedious tasks such as harvesting. Harvesting robots perform harvesting actions after collecting and analysing the information from their surroundings [12]. Hence, robot-assisted tomato harvesting also requires the detection of the target fruit.

Thus, whether for tomato growth monitoring, yield prediction, or for robotic harvesting, tomato fruit detection is a crucial step. In regard to immature on-plant tomato detection in images, there are two main challenges. One is the similarity in colour of an immature tomato to the leaf and vine. For green tomatoes, the aiming pixels cannot be easily segmented using the threshold method or using the calculation of the RGB components [13]. To solve this problem, machine learning was used to mine colour features to help pixel-based classification. By applying a decision tree segmentation model on 15 transformed colour features, immature tomatoes were successfully separated from leaves, stems, and backgrounds [14]. However, it needed blob-based segmentation to further reduce misclassifications due to similar colour. Another method is the use of multispectral sensors to evaluate differences in reflectance to separate fruit from background. For immature green citrus detection, through combining colour and thermal images, an increase in recall from $78.1 \%$ to $90.4 \%$ and an increase in precision from $86.6 \%$ to $95.5 \%$ were found [15]. In addition, by using imagery fused with colour (RGB) and near-infrared (NIR), the F1 scores were increased [16]. Nonetheless, a multispectral camera is costly compared with an RGB camera and is less applicable in large-scale farms.

The other challenge for tomato on plant detection is occlusion issue, i.e., tomatoes overlap or are occluded by foliage and vines. X-means clustering algorithm was thus used to detect the position of each tomato in a multi-fruit blob [14]. However, the algorithm depends on the overexposed region of the tomato surface, which is inconspicuous under natural illumination conditions. Some other researchers attempted to detect citrus on trees by valid contour selection and occlusion recovery [17], but this method requires a relatively long fruit contour length, which is not suitable for highly occluded situations. Then sliding window was used to detect individual tomatoes by extracting the Haar-like features in the sub-window and classifying tomato pixels with the AdaBoost classifier [18], but testing needs to be done to determine the optimal sub-window size and sliding step. Therefore, researchers tried building a region of interest pyramid to adapt to different tomato sizes and then detected tomatoes using histograms of oriented gradients and a pretrained support vector machine (SVM) classifier [19]. Nonetheless, for overlapping or occluded areas, the miss rate was as high as $16 \%$.

To overcome these challenges, deep learning techniques are a good choice even when using RGB cameras. Deep learning can perform classification and make predictions particularly well, being flexible and adaptable for a wide variety of highly complex challenges, such as varying illumination and depth, overlapping, and occlusion. Rahnemoonfar and Sheppard [20] presented a simulation-based deep learning method for fruit counting using a modified version of the Inception-ResNet architecture. Chen et al. [21] used another neural network and linear regression to estimate and sum the number of fruits in blobs detected by a fully convolutional network. Other approaches employ the faster region-based convolutional neural network (R-CNN) model not only to count fruits and vegetables but also to locate their position in the image by means of bounding boxes, which is important for automatic harvesting. Moreover, most of the research works that have incorporated popular deep learning architectures took advantage of transfer learning [22], as sometimes it is not possible to train a network from scratch due to a small training dataset or a complex multitask network. Sa et al. [16] deployed DeepFruits using faster R-CNN with VGG-16 through transfer learning. Bargoti and Underwood [23] also used faster R-CNN with VGG-16 and found that compared to transferring weights between orchards, data augmentation yields significant performance gains. However, previous research relating fruit location achieved high accuracy with a relatively low intersection over union (IoU) threshold compared with the common threshold (IoU $\geq 0.5$ ) in object detection, which increased the error in estimation fruit location and fruit size $[16,23]$. In order to improve the detection accuracy, the deep 
residual learning was one option, as it solved the "vanish gradient" problem and obtained a $28 \%$ relative improvement on the Common Objects in Context (COCO) object detection dataset [24] by reformulating the layers as learning residual functions with reference to the layer inputs. Based on that, some improved deep learning frameworks combined with residual networks appeared, such as Inception-Resnet-v2, which was designed to reap all the benefits of the residual approach while retaining inception architecture's computational efficiency [25]. Therefore, combining faster R-CNN with deep residual networks, e.g., Resnet 50 [24] and Resnet 101 [24], and Inception-Resnet-v2 [25] may achieve high detection accuracy with less detection error.

Tomato-on-plant detection is expected for precision cultivation and for robotic harvesting. However, immature tomatoes that are highly occluded on plants are difficult to detect and locate using traditional image analysis methods, especially under natural illumination conditions. In this paper, we attempted to trained a faster R-CNN model combined with deep residual learning in real tomato cultivation scenes to (1) accurately detect all the visible tomatoes in photo regardless of fruit occlusion and lighting conditions, (2) count the tomato load, and (3) compile a tomato location map and estimate the tomato size along one row in a greenhouse for further yield prediction and robot harvesting.

\section{Materials and Methods}

\subsection{Image Acquisition and Labelling}

The photos were collected in two places, the Seki farm and U-Tokyo farm, by a Canon 60D (Canon Inc., Tokyo, Japan) with a Tamron SP10-24 mm lens (Tamron Co., Ltd., Saitama, Japan). The Seki farm is located in Kiyose City, Tokyo, Japan. The photos from the Seki farm were taken on 19 May 2015 and 22 January 2016. There were nine rows of tomato growing on shelves in a greenhouse, and each row had two sides. On each side, photos were taken $0.75 \mathrm{~m}$ away from the shelf (Figure 1), and the overlap ratio between two adjacent photos was approximately 0.55 . The dimensions of the photos taken on 19 May 2015 were $3456 \times 5184$ pixels and were vertical in direction, and those on 22 January 2016 were $5184 \times 3456$ pixels and were horizontal in direction. The Tanashi greenhouse is located at the U-Tokyo farm, Nishi-Tokyo City, Tokyo, Japan. The tomatoes were planted in pots in a greenhouse. The dimensions of all the photos were $5184 \times 3456$ pixels, and all the photos were taken on 21 January 2016, including 30 photos in the daytime and 32 photos at night. After the photos were collected, they were segmented into three datasets—-training, validation, and testing datasets. See details of the dataset in Table 1.

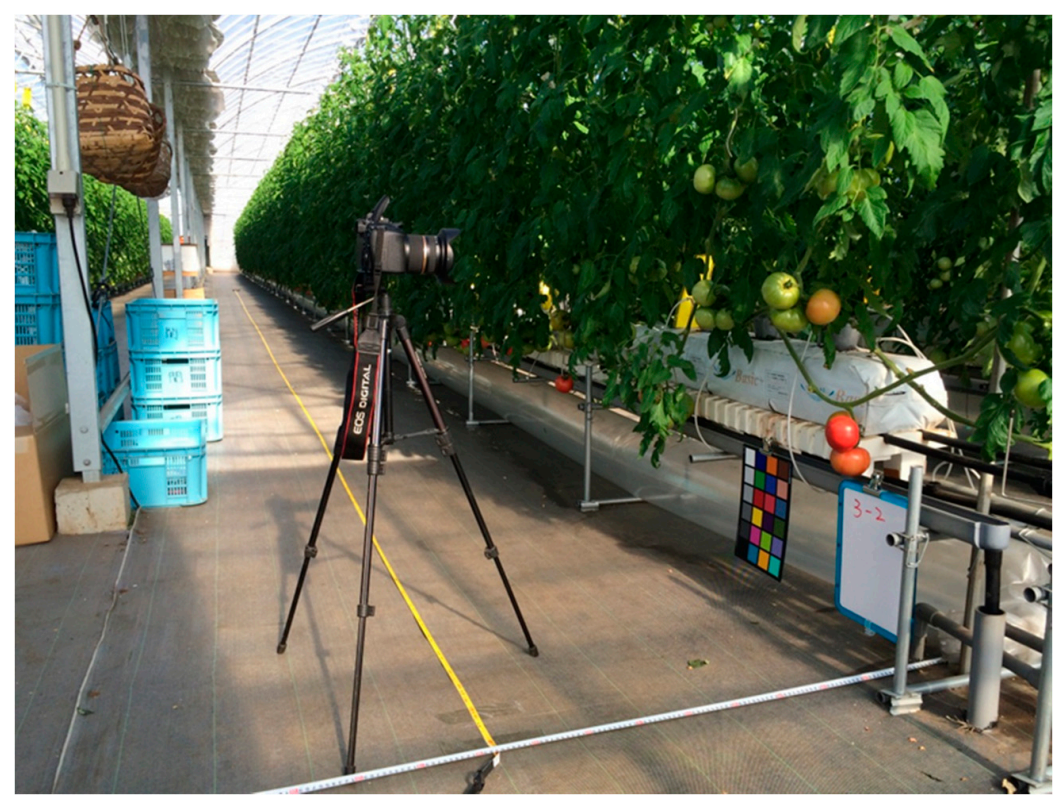

Figure 1. The camera layout for taking tomato photos in the greenhouse of Seki farm. 
Table 1. Details of the dataset.

\begin{tabular}{ccccc}
\hline Location & Date & Time & Photo Number & Photo Size (pixels) \\
\hline \multirow{2}{*}{ Seki farm } & 19 May 2015 & Day & 229 & $3456 \times 5184$ \\
& 22 January 2016 & Day & 349 & $5184 \times 3456$ \\
Tanashi green house & 21 January 2016 & Night & 32 & \\
& & & \\
\hline
\end{tabular}

Tomato labelling was implemented through a web-based interactive labelling tool (http: //fieldphenomics.com/) developed by the U-Tokyo International Phenomics Research Laboratory. In each photo, all the visible tomatoes were labelled by a bounding box, which were required to be tight enough to cover the object, mainly in the range of 20-70 boxes per image. After that, we checked the image annotation three times by different people. Notably, for the highly occluded tomatoes, the bounding boxes were drawn by the supposed shape depending on the visible part (Figure S1). In total, 640 photos with 28,835 tomatoes were manually labelled.

\subsection{Data Pre-Processing}

\subsubsection{Training and Validation Datasets}

The images in the training and validation datasets (Table 2) were used for training the model and accuracy evaluation, respectively. The images were pre-processed by the following four steps: (1) resize the image to 0.5 of its original size; (2) Crop the image into four subimages, considering several factors such as detection accuracy, GPU memory, and convenience of processing; (3) rotate the vertical subimages $(864 \times 1296$ pixels) to horizontal $(1296 \times 864$ pixels) to keep the size of the arrays the same on the two dimensions; and (4) rename the subimages as a digital sequence for processing in TensorFlow.

Table 2. Details of the training and evaluation datasets.

\begin{tabular}{|c|c|c|c|c|c|c|}
\hline Dataset & Location & Date & Time & Photo Number & Subimage Number & Image Size (pixels) \\
\hline \multirow{2}{*}{ Train dataset } & Seki farm & $\begin{array}{c}\text { 19 May } 2015 \\
\text { 22 January } 2016\end{array}$ & Day & \multirow[b]{2}{*}{452} & \multirow[b]{2}{*}{1779} & $864 \times 1296$ \\
\hline & Tanashi green house & 21 January 2016 & $\begin{array}{l}\text { Day } \\
\text { Night }\end{array}$ & & & \multirow{3}{*}{$1296 \times 864$} \\
\hline \multirow[b]{2}{*}{ Evaluation dataset } & Seki farm & 22 January 2016 & Day & & & \\
\hline & Tanashi green house & 21 January 2016 & $\begin{array}{l}\text { Day } \\
\text { Night }\end{array}$ & 129 & 511 & \\
\hline
\end{tabular}

\subsubsection{Test Dataset}

One row of the photos was used as test dataset (Table 3) to test the performance of the model in tomato localization. The images were pre-processed using the following five steps: (1) stitch the adjacent photos into six large images using Image Composite Editor (Microsoft Corporation, Redmond, Washington, USA); (2) pad the image on the left and right sides; (3) resize the image to 0.5 of its original size; (4) crop the image by a fixed size of $1296 \times 864$ pixels, as shown in Figure 2; and (5) rename the subimages as digital sequences for processing in TensorFlow.

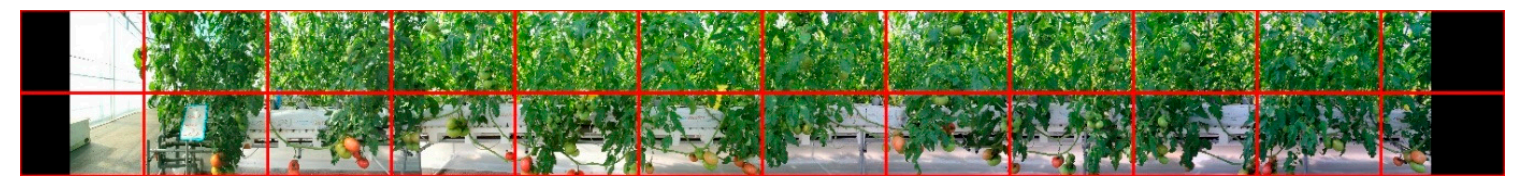

Figure 2. The location of the sub-image in one stitched image of part of the row. Red lines are the outlines of subimages. 
Table 3. Details of the test dataset.

\begin{tabular}{ccccccc}
\hline Dataset & Location & Date & Time & Photo Number & Subimage Number & Image Nize (pixels) \\
\hline Test dataset & Seki farm & 22 January 2016 & Day & 59 & 135 & $1296 \times 864$ \\
\hline
\end{tabular}

\subsection{Tomato Detection Model Generation}

\subsubsection{Train Multiple Tomato Detection Models and Select the Model with the Highest Accuracy}

We chose faster R-CNN as the tomato detection architecture because of its high precision and speed [26]. Faster R-CNN works as follows: (1) run the image through a CNN to obtain a feature map; (2) run the activation map through a separate network, called the region proposal network (RPN), that outputs boxes/regions; and (3) for the boxes/regions from RPN, use several fully connected layers to output class and bounding box coordinates [26].

The tomato detection model was built on a TensorFlow object detection API (https://github.com/ tensorflow/models/tree/master/research/object_detection, Google Inc., Santa Clara, CA, USA) on one workstation with a NVIDIA Tesla P40 graphics card (22919 MB RAM, NVIDIA Corporation, Santa Clara,

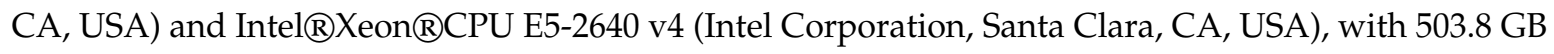
RAM, running a 64-bit Ubuntu 16.04 LTS operation system (Canonical Ltd., London, UK).

The faster R-CNN models were pretrained on the COCO dataset with Resnet-50 [24], Resnet-101 [24], and Inception-Resnet-v2 [25] convolutional neural network models, respectively. Because all of these models have shown high accuracy and speed on the COCO dataset, we wanted to investigate their performance on our dataset. With 1779 subimages as training data, 511 subimages as validation data, a learning rate of 0.00003 , and random horizontal flip as the data augmentation method, these three models were trained by transfer learning and obtained an average precision (AP) of intersection of union (IoU) $\geq 0.5$ in 100 epochs on the validation dataset. Other hyperparameters used the default settings in the faster R-CNN configure file. By comparing the $\mathrm{AP}$, we selected the model with the highest accuracy as tomato detection model.

\subsubsection{Detect Tomatoes Using the Selected Model on the Test Dataset}

The hyperparameters such as learning rate has been fine tuned to increase the accuracy of the validation data. To test whether the model overfit, the model was applied to the test dataset. The detection was performed by the following five steps: (1) export the TensorFlow graph of the specified checkpoint of the highest AP in the selected model; (2) infer detections from the test dataset with the exported TensorFlow graph with non-maximal suppression threshold of 0.6 ; (3) evaluate the detections with manually labelled tomatoes as reference and obtain the AP of IoU $\geq 0.5$; (4) filter out the boxes with confidence scores below 0.5 ; and (5) record the detected tomato number and locations.

\subsection{Evaluation Metrics}

The average precision with IoU thresholds of 0.5 was used to quantify the model accuracy. This metric is popular for measuring the accuracy of object detectors, as it balances the performances of precision and recall. The average precision could be calculated by the area under the precision-recall curve. Precision is calculated by Equation (1), and recall is calculated by Equation (2), where TP is the number of true positives, $F P$ is the number of false positives, and $F N$ is the number of false negatives. Both of these parameters are calculated on all the boxes by rank according to the descending predicted confidence.

$$
\begin{gathered}
\text { Precision }=\frac{T P}{T P+F P} \\
\text { Recall }=\frac{T P}{T P+F N}
\end{gathered}
$$


In this research, we used COCO detection metrics to calculate the average precision. The COCO metrics are the official detection metrics used to score the COCO competition (http://cocodataset.org/) and can report statistics such as AP at IoU thresholds of 0.5 .

\subsection{Tomato Localization}

Then, tomatoes detected in subimages was composed together and located in the stitched image. The tomato localization was performed by the following five steps: (1) rename the detected sub-image to its original name for linking to the original image; (2) put the detected sub-image to the original location of the stitched image; (3) recalculate the box coordinates in the stitched image and label the boxes on the edge of the subimage; (4) Merge the boxes on the seams by recognizing the split parts using the box distance and shape similarity (ratio of the side length in both directions); and (5) calculate the number of tomatoes in each stitched image and outline the detected boxes.

\subsection{Tomato Size Estimation}

Finally, tomato sizes in images including width, height, and aspect ratio were determined by the bounding box merged in the location map. The tomato width and height were represented by the bounding box width and height, and the aspect ratio was calculated by the ratio of the width to the height.

\section{Results}

\subsection{Accuracy Analysis of Deep Learning Models}

There were three deep learning models trained with different deep convolutional neutral networks, i.e., Resnet 50, Resnet 101, and Inception-Resnet-v2. Running the models on the validation images yielded the change in average precision with the epoch (Figure 3). It was observed that all the models obtained the highest accuracy at approximately 10 epochs, and then the accuracy decreased. This may be caused by overfitting, as the size of training dataset was quite small compared with the COCO dataset. Among the models, the deep learning model with Resnet 101 achieved the highest AP of 0.82 , so this model was selected as the tomato detection model.

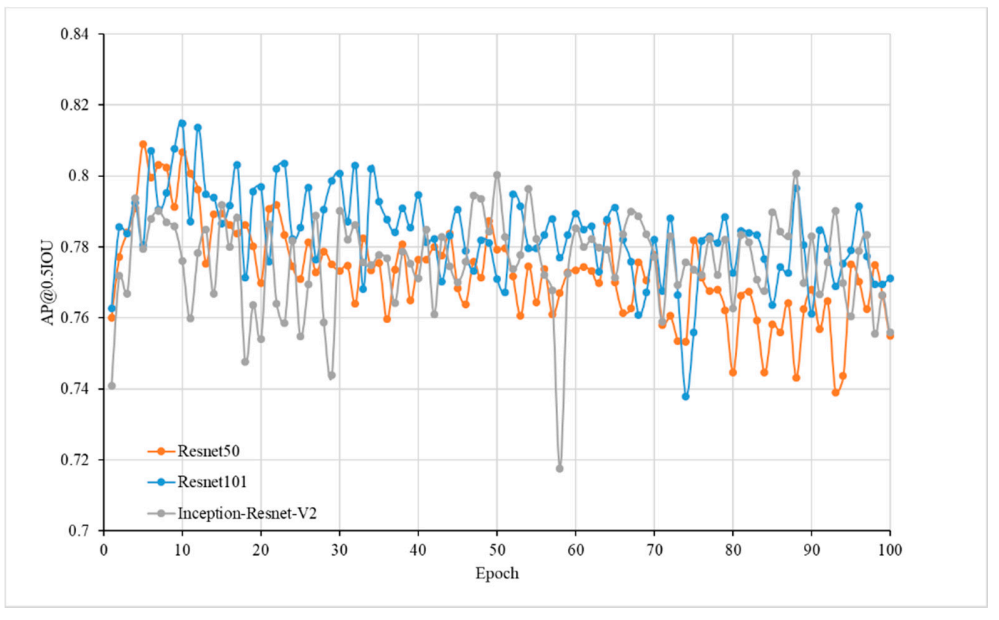

Figure 3. Accuracy of the tomato detection model.

Then, the model was applied on the test dataset and achieved the AP of 0.87. Then, the detected results were further analysed with the distribution of true positive, false positive, and false negative at an IoU threshold of 0.5 . True positive means that a box was detected as a tomato and overlapped with one manually labelled tomato box, and the interception area was greater than 0.5 of the union of their areas. False positive means a box was detected as a tomato, but its IoU was less than 0.5 
with any manually labelled tomato box. False negative means a box was not detected as a tomato, but its IoU was greater than 0.5 with one or more manually labelled tomato boxes. Therefore, the false positive number is the number of detected tomatoes that are not tomatoes or that do not meet the IoU requirements, and the false negative number is the number of missed tomatoes or those that were not accurately located.

We counted the number of true positives and false positives based on the detected tomato box and its scores. Figure $4 \mathrm{~A}$ shows that as the score increased, the percentage of false positives decreased. At the same time (Figure $4 \mathrm{~A}$ ), the percentage of true positives increased, which means that more detected tomato boxes met the requirement of IoU. The relative frequency distribution (Figure 4B) showed that more than $80 \%$ of the false positives had scores less than 0.1 , and more than $80 \%$ of the true positives had scores not less than 0.9. As shown in Figure 5A, when the score was low, there were many overlapping boxes with poor location precision. This may lead to many false positive detections at $\mathrm{IoU} \geq 0.5$. Comparing the tomato sizes in Figures $5 \mathrm{C}$ and $5 \mathrm{D}$ indicated that large size tomatoes tended to have high scores. However, there was a small portion of manually labelled tomatoes with scores less than 0.2 .
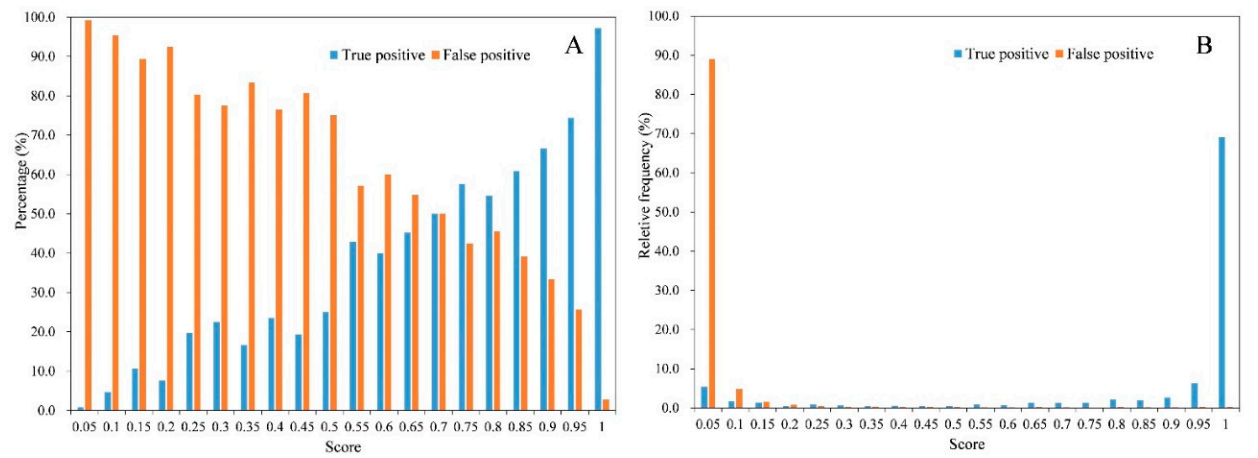

Figure 4. Distribution of true positives and false positives with scores by applying the tomato detection model on the test dataset. (A) Percentage change of true positives and false positives with scores; (B) relative frequency change of true positives and false positives.

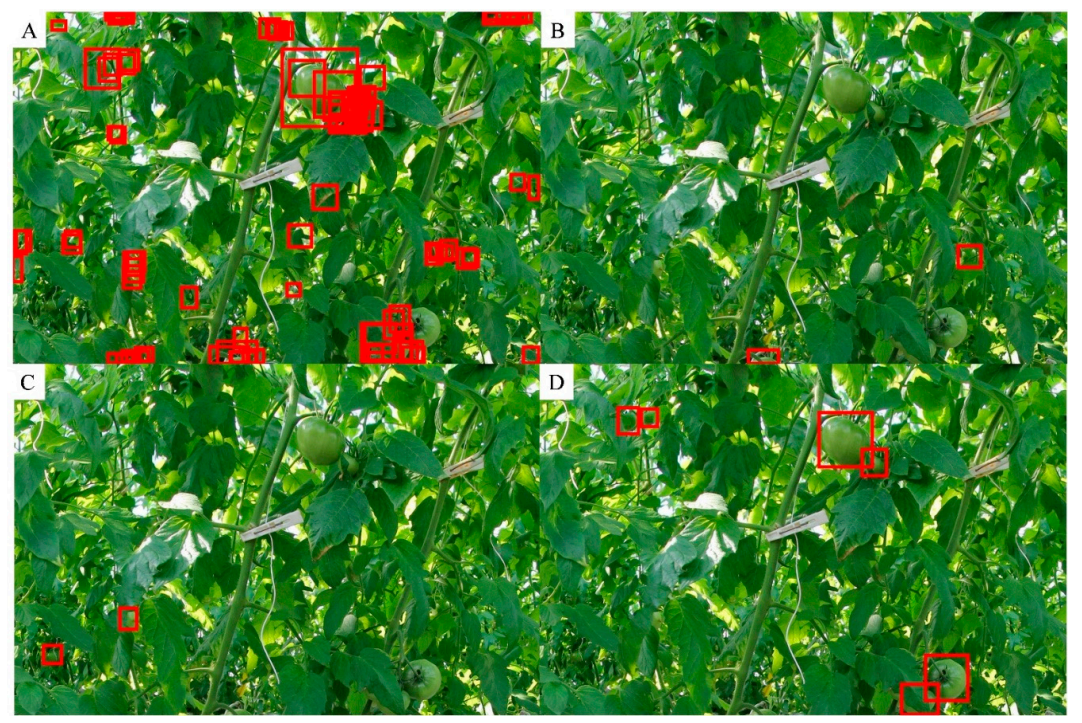

Figure 5. Examples of detected tomatoes with different scores in the test dataset. Subfigure (A) shows boxes with scores $\leq 0.3$, (B) shows boxes with $0.3<$ scores $\leq 0.5$, (C) shows boxes with $0.5<$ scores $<0.7$, and (D) shows boxes with scores $>0.7$. 


\subsection{Tomato Counting Assessment}

Tomato counting is the basic step for yield estimation $[9,21]$. Through the application of the selected deep learning model to the test dataset, tomatoes were detected, and the boxes of tomatoes in each subimage were labelled (Figure 6A,C). According to the distribution of true positives (Figure 4), the detected tomatoes were filtered by score. Then, only the tomatoes with scores $\geq 0.5$ were retained (Figure 6B,D), and the number of tomatoes in each subimage was recorded. Correlation analysis of the number of labelled and detected tomatoes per subimage showed a high coefficient of determination $\left(\mathrm{R}^{2}=0.87\right)$. However, the number was slightly underestimated when the tomato number was greater than 20 per subimage, as shown in Figure 7. This may be caused by some tomatoes with low scores (less than 0.5), which were filtered out. One example is the tomato in the image shown in the blue box of Figure 6D, of which less than a quarter was visible and was quite small, but it has been manually labelled.

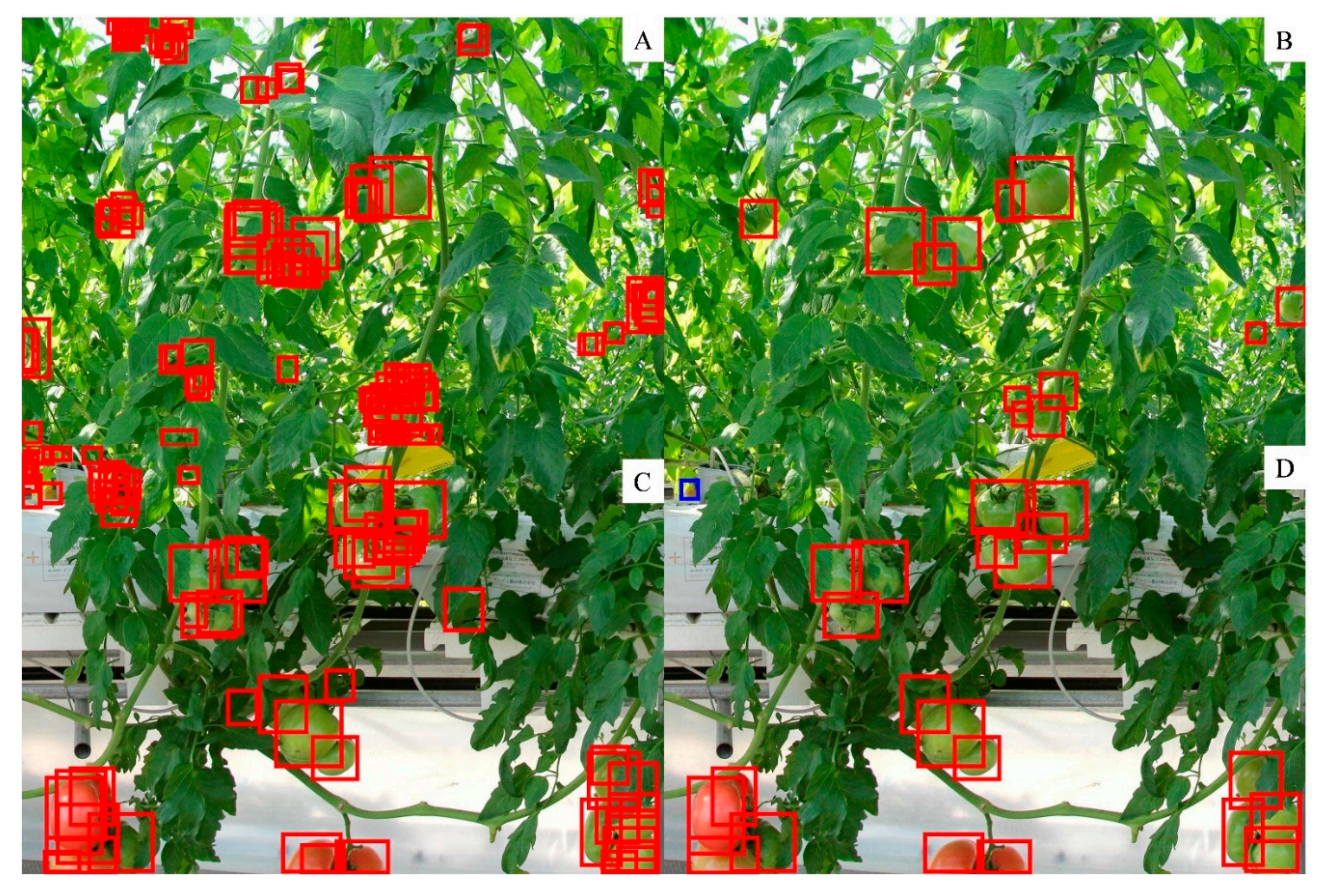

Figure 6. Example of all detected tomatoes $(\mathbf{A}, \mathbf{C})$ and filtered detected tomatoes with a score $\geq 0.5$ in the test dataset $(\mathbf{B}, \mathbf{D})$.

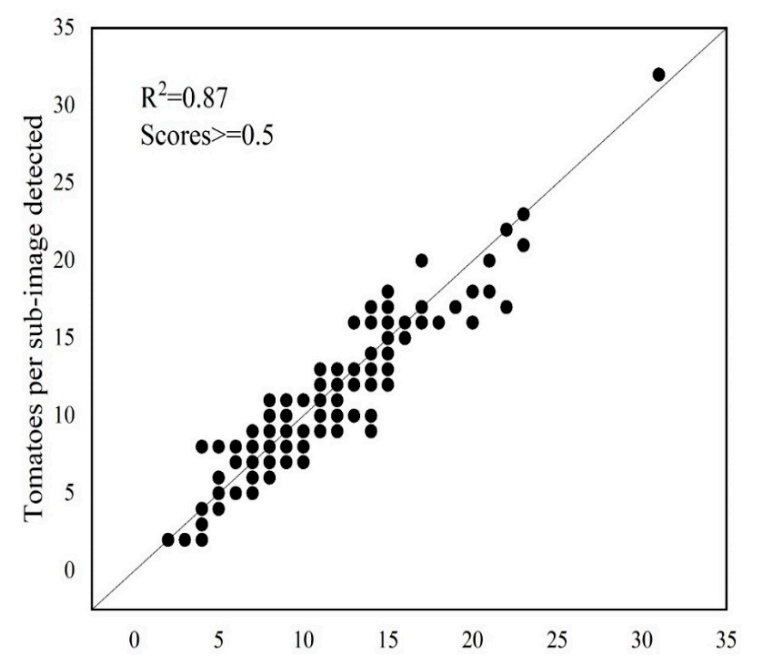

Figure 7. Correlation between labelled and detected tomatoes per subimage in the test dataset. 


\subsection{Tomato Localization for the Whole Cultivation Bed}

Yield mapping is quite important for monitoring the growth of tomatoes, providing information on spatial variation on which to base agronomic decisions [7]. Precise tomato detection allows for the generation of yield maps and could also be an assistant tool for robotic harvesting. After the tomatoes in the subimages were detected and labelled, the tomatoes were transferred to the stitched image of one cultivation row in the green house, and the segmented box on the seaming line was merged. In total, 1422 tomatoes were detected and located along this row. Figure 8A shows the location map for tomatoes in one row in the greenhouse of the Seki farm. By enlarging some zones in Figure 8A, it shows that the red tomatoes were all near the bottom of the shelf, and the green tomatoes were mainly in the upper region (Figure 8B,C).

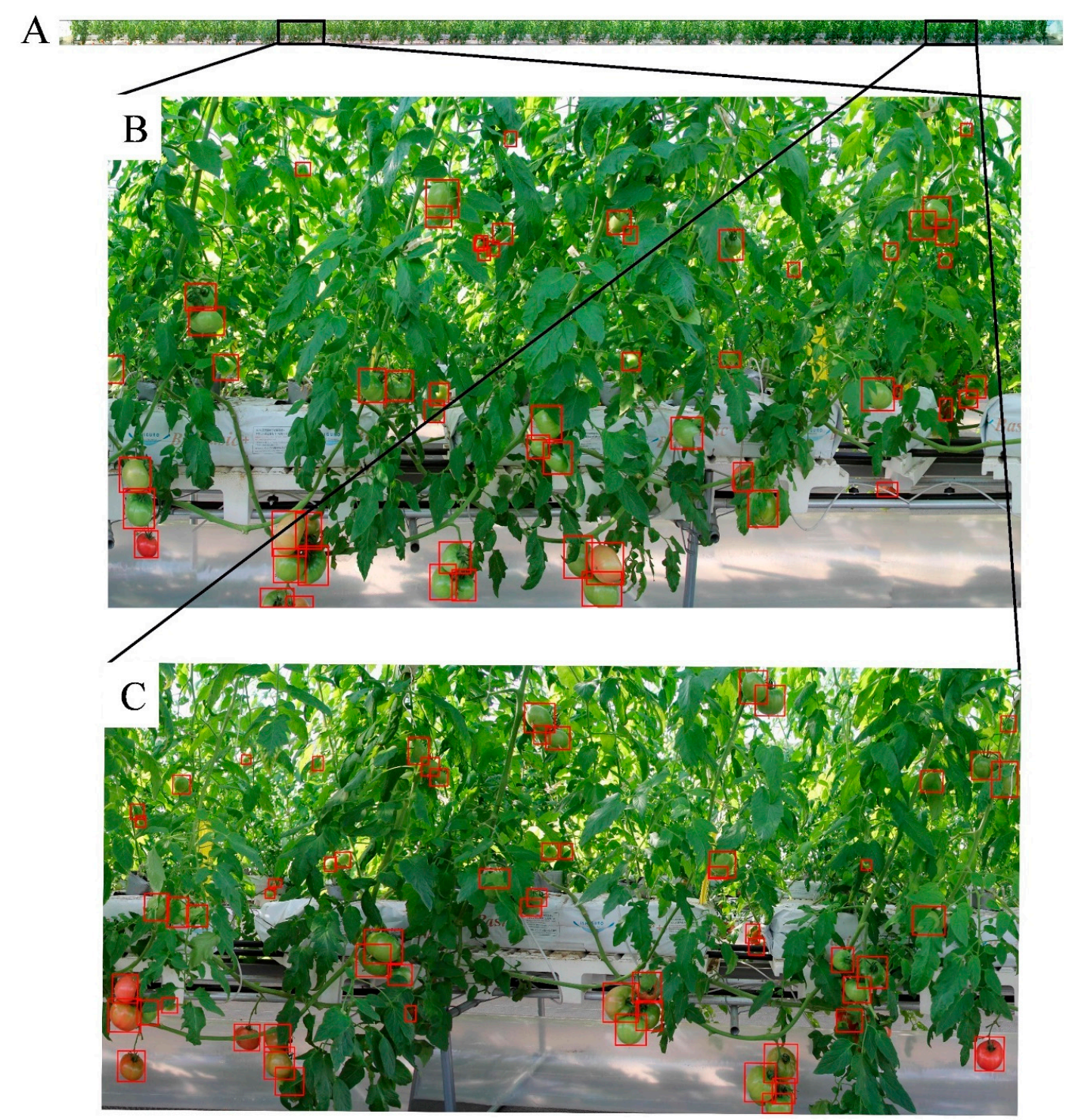

Figure 8. Detected bounding boxes of the tomatoes in the stitched image. (A) is the location map for tomatoes in one row; $(\mathbf{B}, \mathbf{C})$ are two enlarged zones in $(\mathbf{A})$ for clear illustration.

\subsection{Tomato Size Estimation}

Tomato size estimation is of great significance for yield estimation [7,27] and prediction $[8,10]$. On-tree estimation of fruit size is useful for the prediction of maturity and harvest time $[8,10]$, and estimation of fruit size together with fruit number allows estimation of fruit weight ('yield') $[7,27]$. 
By using the faster R-CNN models with Resnet-101, the final detection of tomato showed precise detection. Tomato length, width, and aspect ratio in an image can be determined based on the bounding box. Taking the tomato merged in one row (see details in Section 2.5) as an example, the size distribution (Figure $9 \mathrm{~A}$ ) showed that about $16.53 \%$ and $13.78 \%$ of the tomatoes had a width and height less than 50 pixels, respectively. The aspect ratio (Figure 9B) showed that about $89 \%$ tomatoes had an aspect ratio in the range of 0.67 to 1.27 , and about $33 \%$ tomatoes had an aspect ratio in the range of 0.97 to 1.17. The closer the aspect ratio value was to 1 , the closer the shape of tomato was to a circle.
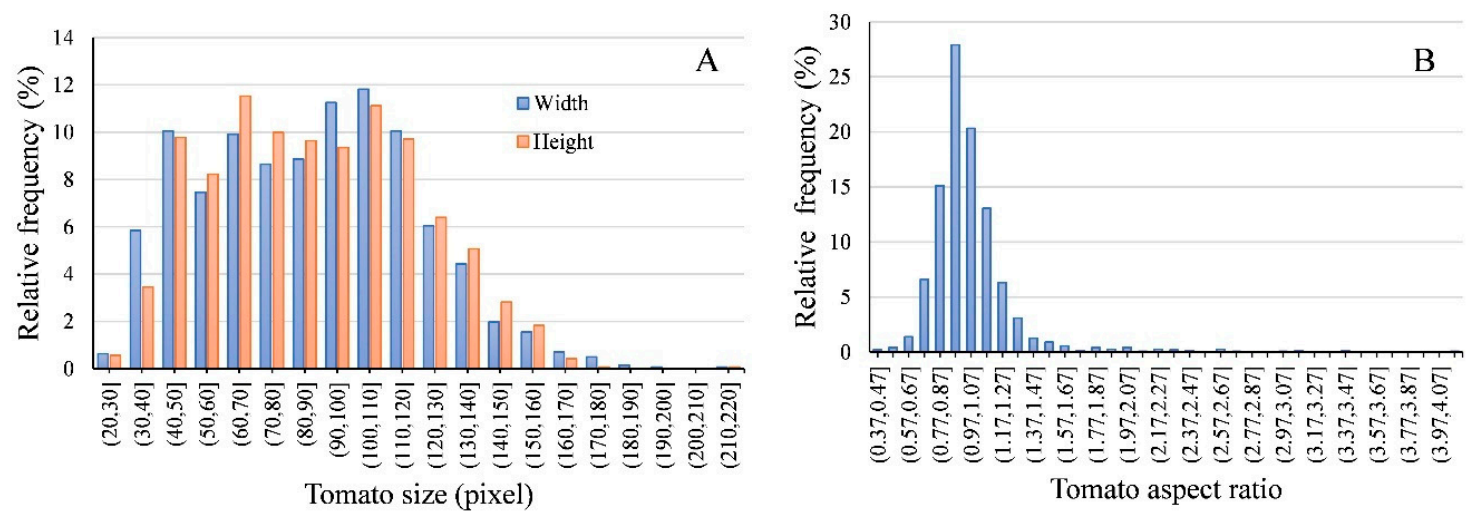

Figure 9. Relative frequency change of the tomatoes size (A) and aspect ratio (B) in images. Tomato size is represented by box width and box height. Tomato aspect ratio is calculated by width/height.

\section{Discussion}

\subsection{Accuracy Comparison with Other Tomato-on-Plant Detection Techniques Using RGB Images}

Using the faster R-CNN models with Resnet-101, the tomato detection model showed relatively high precision on the test dataset. To compare with previous tomato detection performance, the method and the best result of each paper are listed in Table 4. Following the suggestion by Koirala [7], the F1 score of the test detection in this research was calculated for comparison (see Figure S2). When available, the F1 score of other researches were recorded. Some previous researches lack the F1 score recording or precision-recall curve for determining the F1 score $[13,14,18,28]$, but from their accuracy descriptions, the accuracy of this research were higher than most machine learning method. The research [19] got a high F1 score for mature tomato detection, which was less difficult than for green tomatoes. However, when compared with other deep learning approaches $[29,30]$, this method showed lower accuracy. On one hand, this was due to the lower resolution or smaller size of tomatoes in our image, as we aimed to compile a tomato layout map. On the other hand, both of their studies optimised the non-maximum suppression with IoU, which inspired us for future work.

Table 4. Scientific reports in tomato on plant detection based on colour (RGB) images. The best result of each paper is shown. When available, the F1 score is recorded; otherwise, the validation metric used by the authors is included.

\begin{tabular}{ccc}
\hline Author & Method & Accuracy \\
\hline $\begin{array}{c}\text { Schillaci et al., 2012 [28] } \\
\text { Khoshroo et al., 2014 [13] }\end{array}$ & $\begin{array}{c}\text { Scanning window with support vector machine } \\
\text { Colour analysis and region growing } \\
\text { Pixel-based segmentation, blob-based } \\
\text { segmentation, X-means clustering }\end{array}$ & $\begin{array}{c}\text { Twenty true positives against 26 false positive } \\
\text { Overall classification accuracy: } 82.38 \%\end{array}$ \\
& AdaBoost classifier and colour analysis & Recall: 0.8, precision: 0.88 \\
Yamamoto et al., 2014 [14] & True positives rate: $96.5 \%$ \\
Zhao et al., 2016 [18] & Faster R-CNN with Resnet 50 & Missing (False negative) rate: $3.5 \%$ \\
Sun et al., 2018 [29] & Machine learning and colour analysis & mAP (green and red tomatoes): $90.9 \%$ \\
Liu et al., 2019 [19] & Yolo-tomato & F1 score: $92.15 \%$ \\
Liu et al., 2020 [30] & Faster R-CNN with Resnet 101 & F1 score: $93.91 \%$, AP: $96.40 \%$ \\
This paper & & F1 score: $83.67 \%$, AP: $87.83 \%$ \\
\hline
\end{tabular}




\subsection{Error Analysis}

\subsubsection{Overfitting}

Overfitting is a common problem in machine learning. According to observations of the AP with the epoch (Figure 10), the faster R-CNN model with Resnet 101 showed overfitting after epoch over 10. One reason may be the size of training dataset was quite small which consisted of 1779 subimages $(864 \times 1296$ pixels), as the model was pretrained on the COCO dataset, which consisted of 118,287 images and 80 classes for training (https://www.tensorflow.org/datasets/catalog/coco). Enlarging the training data size and diversity may help to solve this problem. Data augmentation techniques [31] could artificially enlarge both the number and the variety of training images and have been shown to yield significant performance gains [23]. In this research, only random horizontal flip was used as the data augmentation method, and more data argumentation methods will be used to get a better training model in the future.

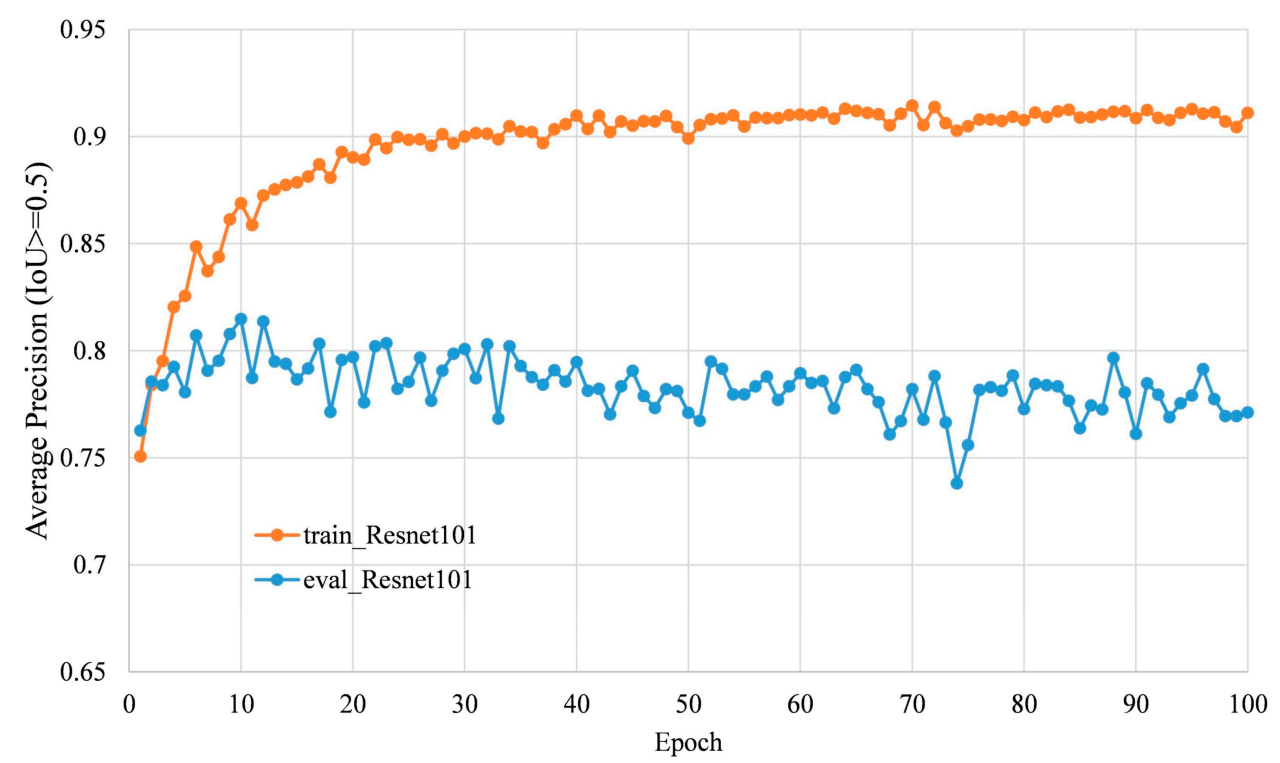

Figure 10. Change in average precision (AP) at an intersection of union (IoU) threshold of 0.5 of training data and validation data using Resnet 101 with the epoch.

\subsubsection{Manual Labelling}

Manual labelling served as ground truth in the accuracy analysis. For precise fruit detection, ground truth boxes should be tight enough to cover the object and some of the background around the object perimeter [32]. However, we cannot ensure that all the visible tomatoes were labelled and all the placement for boxes were appropriate, even though we checked the images three times. For the same tomato, different people will label the tomato with a box of different sizes and locations. This causes errors in tomato training and detection. Several efficient labelling methods have been developed [33-35]. Use of these methods not only saves time spent labelling but also uses less labour, making it possible to standardize the method of box placement.

\subsubsection{Influence of Tomato Size on Determination}

The results (Figure 6D) showed that very small tomatoes (in image size) may have a higher possibility regarding false or missing detection. Other than less information increasing the difficulty in recognition, it was partly due to the criterion for determining truth positive. The detection was recognized as true positive only if it has enough overlap with the manually label box measured by IoU. Based on the IoU calculation equation, the smaller the object is, the lower the IoU value when offset in pixels. As shown in Figure 11, the IoU of the left boxes (Figure 11A) is 0.53 , and the IoU of the right 
boxes (Figure 11B) is 0.47 . This means that for very small tomatoes, a small misplacement of the box causes a low IoU. Hence, even small errors in the detection of smaller fruit caused these fruits to be registered as false positives [23]. As Figure 12 showed, by calculating the moving median value of every 51 true positive boxes and false positive boxes, respectively, the box area of true positive was a little larger than false positive at all the scores. The box area of $28.99 \%$ of the false positive boxes was smaller than 2000 pixels, and $81.16 \%$ of the false positive boxes was smaller than 5000 pixels, while that of only $5.59 \%$ and $28.33 \%$ of the true positive, respectively (Table 5). Therefore, the detector seems to work better for tomatoes with a bounding box area large than 2000 pixels and could largely reduce false positives for tomatoes with a bounding box area large than 5000 pixels. This issue could be solved by increasing the image resolution in the future.

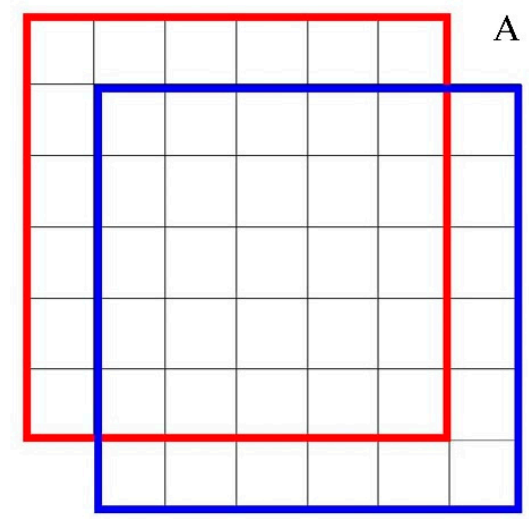

B

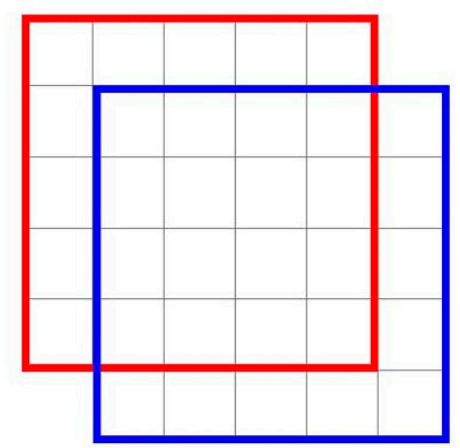

Figure 11. Illustration of the IoU value difference for the same offset in pixels but for different size objects. The IoU of the left boxes (A) is 0.53 , and the IoU of right the boxes $(\mathbf{B})$ is 0.47 . Although both of them has the same offset (one pixel) in the same direction, the left detection is true positive while the right one is false positive with IoU threshold of 0.5 .

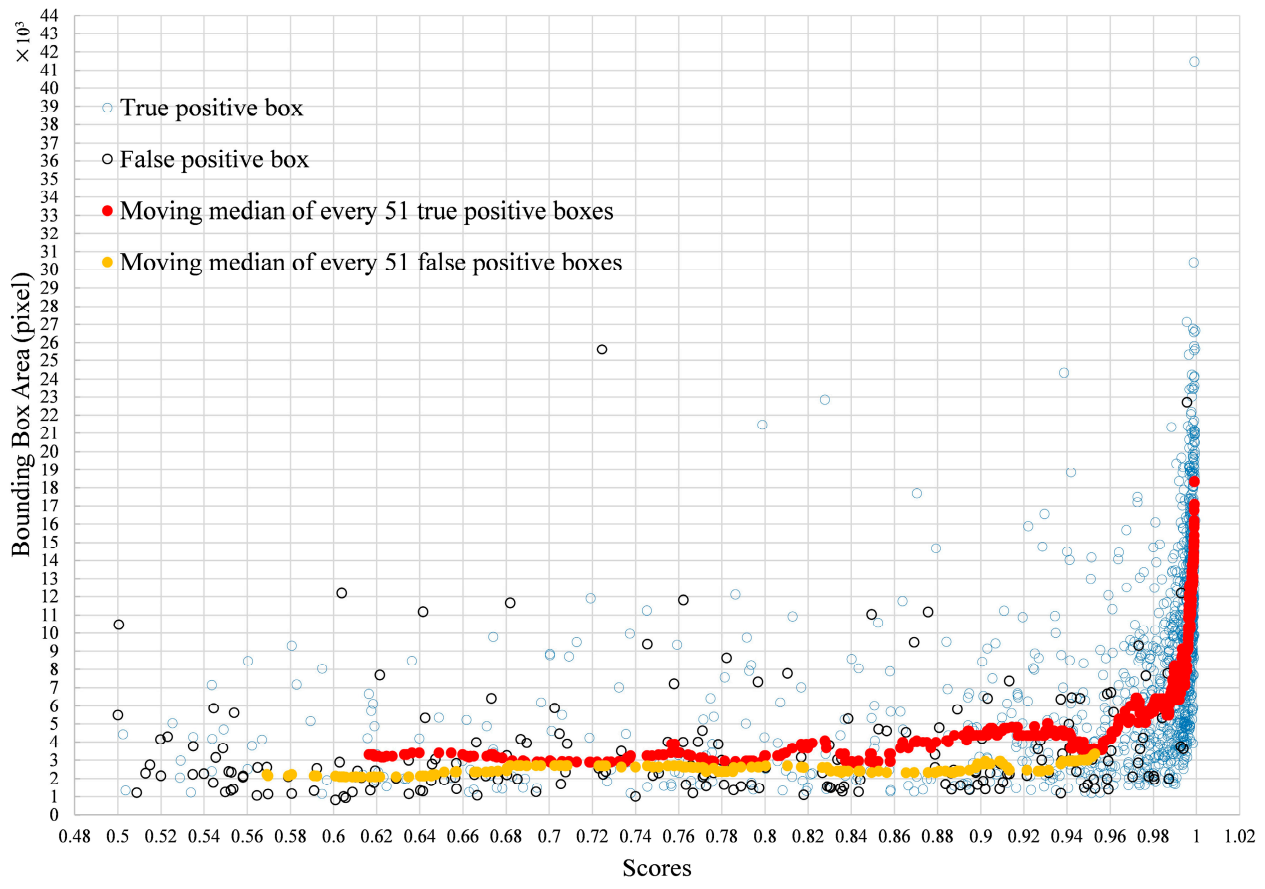

Figure 12. Area distribution of detected true positive boxes and false positive boxes with the scores, and their moving median value with the scores. 
Table 5. Cumulative relative frequency of true positive and false positive boxes of box area.

\begin{tabular}{ccc}
\hline Box Area (Pixel) & True Positive Boxes & False Positive Boxes \\
\hline$\leq 1000$ & $0.00 \%$ & $0.97 \%$ \\
$\leq 2000$ & $5.59 \%$ & $28.99 \%$ \\
$\leq 3000$ & $13.97 \%$ & $59.42 \%$ \\
$\leq 4000$ & $20.83 \%$ & $72.95 \%$ \\
$\leq 5000$ & $28.33 \%$ & $81.16 \%$ \\
\hline
\end{tabular}

\subsection{Limitations}

\subsubsection{Long Training Time}

We used transfer learning for tomato detection, which has some shortcomings in training time compared with other machine learning methods, e.g., support vector machine and random forest methods. However, compared with the training time, the testing time cost of detection per image is more important for application. We tested the model on one mini-size ZBOX computer (ZOTAC, Shenzhen, China) that was equipped with an Intel $®$ Core $^{\mathrm{TM}}$ i5-7500T CPU (Intel Corporation, Santa Clara, CA, USA), NVIDIA GeForce GTX 1060 (NVIDIA Corporation, Santa Clara, CA, USA) and 64-bit Ubuntu 16.04 operation system (Canonical Ltd., London, UK). The model required $0.37 \mathrm{~s}$ to detect tomatoes per image, which is acceptable for real-time detection. In addition, if we take into account the time needed to manually design filters and extract features, "the time used for annotating images and training the $\mathrm{CNN}$ becomes almost negligible" [36].

\subsubsection{Only Visible Tomatoes}

A fruit load estimation relies on the assessment of the total number of fruits per tree, not the number of fruit visible in an image [32]. As this method is based on RGB images, it could only detect the tomatoes visible in the image. If the tomatoes are entirely shaded by leaves or other tomatoes, then they cannot be counted. Actually, the tomato growing "wall" is 3D, but we can only see two dimensions from the image. In the future, by increasing the number of image viewpoints by taking photos on both sides of the row and merging the tomatoes detected from both sides of the row, we could reduce the influence of the nonvisible and get the real tomato counts.

\subsubsection{Tomato Size Estimation in Images}

The tomato size measured in real-world dimensions such as in centimetres is more important for estimating tomato yield. However, in this research, we only used one camera and obtained 2D images, while the tomatoes in the same image has different distances to the camera considering that the tomato was not located on the same plane and the effects of camera projection. This means that we should not use a fix conversion parameter from image size to real size. In the future, we will consider using a multi-vision camera to generate depth information to get the distance of each object to the camera, and this would assist in estimating tomato real size as a conversion reference from tomato image size.

\subsection{Perspectives on Ripeness Estimation and Yield Prediction}

As each tomato was labelled by a bounding box, the ripeness of the tomatoes could be estimated. The tomatoes could be cropped by a bounding box and compared with reference tomato images. Then, by using customized bag of colour layout features, their similarity could be assessed. All the detected tomatoes had a similarity score in the range of zero to one, with more similarity to the referential tomato for a higher score. There is an example shown in Figure 13. 


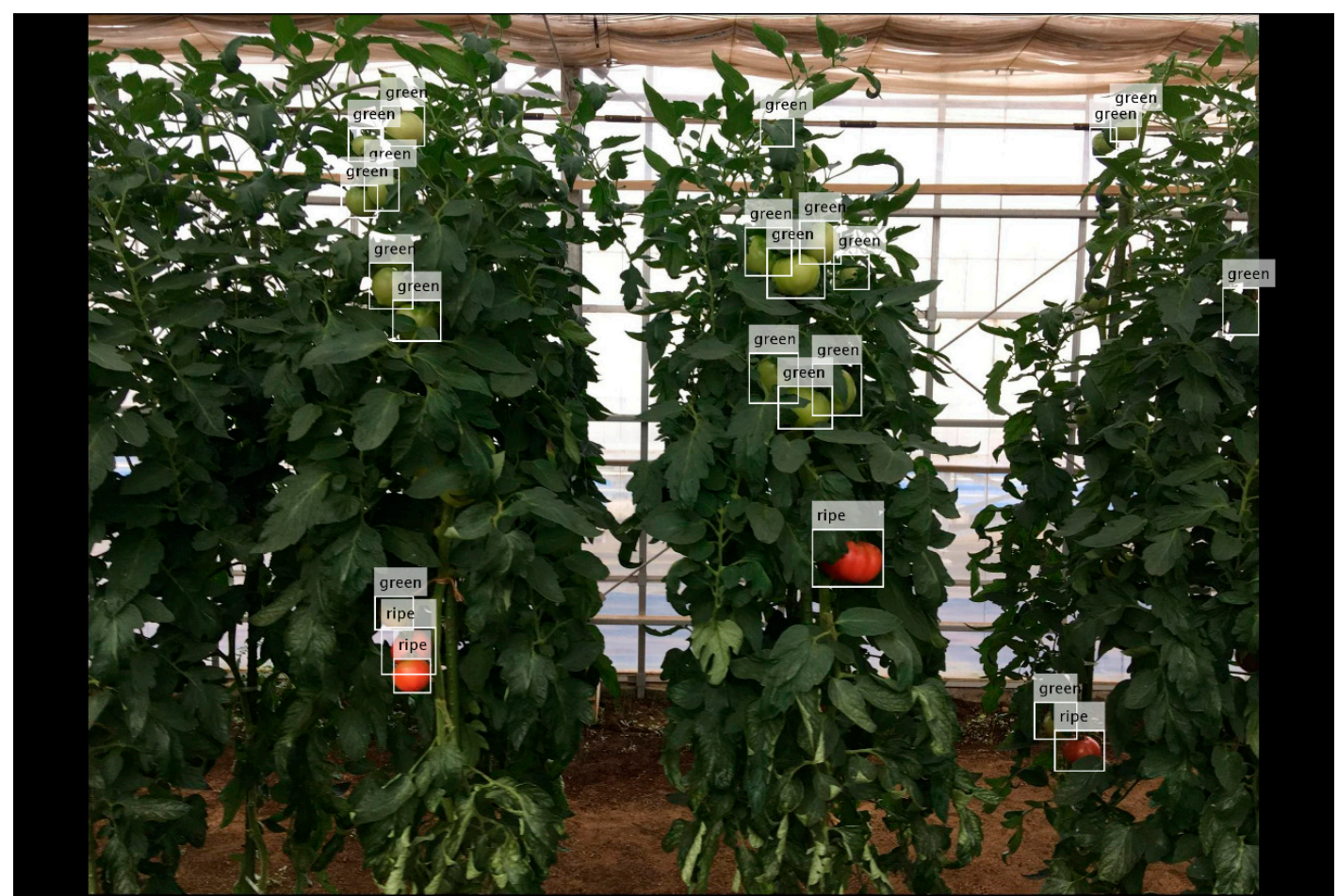

Figure 13. Application of tomato detection in ripeness estimation. The tags labelled ripe or immature tomatoes.

Deep learning has yet to be applied to for prediction of fruit load per tree and per orchard [32]. However, in crop yield prediction such as corn [37] or soybean [38], it already has some attempts. By combining time series of tomato growth data (i.e., tomato size, number and ripeness status) and the environmental data (e.g., weather, soil data) collected in internet of things enabled greenhouse [11], as well as diseases detection [39], using deep learning to build a long short-term memory model with convolution neutral network has great potential in yield prediction.

\section{Conclusions}

In this paper, we trained three faster R-CNN models combined with three deep residual networks (i.e., Resnet 50, Resnet 101, and Inception-Resnet-v2) for tomato detection. The tomato detection model using faster R-CNN with Resnet 101 achieved the highest average precision and was selected for tomato detection. It got average precision of $87.83 \%$ ( $\mathrm{IoU} \geq 0.5$ ) on test dataset, showing good accuracy for detecting highly occluded immature tomatoes on plants in real cultivation scenes. For tomato counting, it received a high coefficient of determination $\left(R^{2}=0.87\right)$ with tomato manually labelled considering more than $10 \%$ quite small tomatoes (size $<50$ pixels). By locating the tomatoes and merging the split tomatoes, 1422 tomatoes were detected and located along one tomato cultivation row in the greenhouse, and their sizes were estimated based on the bounding box. By tomato detection, counting, location, and size estimation, this method shows great potential for ripeness and yield prediction.

Supplementary Materials: The following are available online at http:/www.mdpi.com/1424-8220/20/10/2984/s1, Figure S1. Example of tomato bounding box labelling by a web-based interactive labelling tool (http://fieldphenomics. $\mathrm{com} /$ ); Figure S2. Precision-recall curve of the tomato detection model on test dataset.

Author Contributions: Conceptualization, W.G.; Data curation, T.-S.C.; Formal analysis, Y.M. and S.N.; Funding acquisition, S.N.; Investigation, T.-S.C. and W.G.; Methodology, Y.M.; Supervision, W.G.; Validation, Y.M.; Writing-original draft, Y.M.; Writing—review and editing, Yue Mu, T.-S.C., S.N., and W.G. All authors have read and agreed to the published version of the manuscript.

Funding: The CREST Program (JPMJCR1512) of the Japan Science and Technology Agency; the Fundamental Research Funds for the Central Universities (KYRC202002) of China; the SICORP Program "Data Science-based Farming Support System for Sustainable Crop Production under Climatic Change" of the Japan Science and Technology Agency. 
Acknowledgments: We thank Kenichi Seki for support in the field experiment and useful suggestions. This study was partially funded by the CREST Program (JPMJCR1512) of the Japan Science and Technology Agency, the Fundamental Research Funds for the Central Universities (KYRC202002) of China, and the SICORP Program "Data Science-based Farming Support System for Sustainable Crop Production under Climatic Change" of the Japan Science and Technology Agency.

Conflicts of Interest: The authors declare that they have no conflict of interest.

\section{References}

1. Peixoto, J.V.M.; Neto, C.M.; Campos, L.F.; Dourado, W.D.S.; Nogueira, A.P.; Nascimento, A.D. Industrial tomato lines: Morphological properties and productivity. Genet. Mol. Res. 2017, 16, 1-15. [CrossRef]

2. Food and Agriculture Organization of the United Nations. FAOSTAT. Available online: http://www.fao.org/ faostat/en/\#data/QC (accessed on 29 October 2019).

3. Li, Y.; Wang, H.; Zhang, Y.; Martin, C. Can the world's favorite fruit, tomato, provide an effective biosynthetic chassis for high-value metabolites? Plant Cell Rep. 2018, 37, 1443-1450. [CrossRef] [PubMed]

4. Food and Agriculture Organization of the United Nations. Tomato | Land \& Water. Available online: http://www.fao.org/land-water/databases-and-software/crop-information/tomato/en/ (accessed on 29 October 2019).

5. Sinivasan, R. Safer Tomato Production Methods: A Field Guide for Soil Fertility and Pest Management; AVRDC-The World Vegetable Center: Shanhua, Taiwan, 2010; Volume 10-740, ISBN 92-9058-182-4.

6. Rutledge, A.D. Commercial Greenhouse Tomato Production. Available online: https://extension.tennessee. edu/publications/Documents/pb1609.pdf (accessed on 16 April 2020).

7. Koirala, A.; Walsh, K.B.; Wang, Z.; McCarthy, C. Deep learning-Method overview and review of use for fruit detection and yield estimation. Comput. Electron. Agric. 2019, 162, 219-234. [CrossRef]

8. Austin, P. A Compartment Model of the Effect of Early-Season Temperatures on Potential Size and Growth of "Delicious" Apple Fruits. Ann. Bot. 1999, 83, 129-143. [CrossRef]

9. Malik, Z.; Ziauddin, S.; Shahid, A.R.; Safi, A. Detection and Counting of On-Tree Citrus Fruit for Crop Yield Estimation. IJACSA Int. J. Adv. Comput. Sci. Appl. 2016, 7. [CrossRef]

10. Jha, S.N.; Kingsly, A.R.P.; Chopra, S. Physical and mechanical properties of mango during growth and storage for determination of maturity. J. Food Eng. 2006, 72, 73-76. [CrossRef]

11. Somov, A.; Shadrin, D.; Fastovets, I.; Nikitin, A.; Matveev, S.; Seledets, I.; Hrinchuk, O. Pervasive Agriculture: IoT-Enabled Greenhouse for Plant Growth Control. IEEE Pervasive Comput. 2018, 17, 65-75. [CrossRef]

12. Ling, X.; Zhao, Y.; Gong, L.; Liu, C.; Wang, T. Dual-arm cooperation and implementing for robotic harvesting tomato using binocular vision. Robot. Auton. Syst. 2019, 114, 134-143. [CrossRef]

13. Khoshroo, A.; Arefi, A.; Khodaei, J. Detection of red tomato on plants using image processing techniques. Agric. Commun. 2014, 2, 9-15.

14. Yamamoto, K.; Guo, W.; Yoshioka, Y.; Ninomiya, S. On plant detection of intact tomato fruits using image analysis and machine learning methods. Sensors 2014, 14, 12191-12206. [CrossRef]

15. Gan, H.; Lee, W.S.; Alchanatis, V.; Ehsani, R.; Schueller, J.K. Immature green citrus fruit detection using color and thermal images. Comput. Electron. Agric. 2018, 152, 117-125. [CrossRef]

16. Sa, I.; Ge, Z.; Dayoub, F.; Upcroft, B.; Perez, T.; McCool, C. DeepFruits: A fruit detection system using deep neural networks. Sensors 2016, 16, 1222. [CrossRef]

17. Lu, J.; Sang, N. Detecting citrus fruits and occlusion recovery under natural illumination conditions. Comput. Electron. Agric. 2015, 110, 121-130. [CrossRef]

18. Zhao, Y.; Gong, L.; Zhou, B.; Huang, Y.; Liu, C. Detecting tomatoes in greenhouse scenes by combining AdaBoost classifier and colour analysis. Biosyst. Eng. 2016, 148, 127-137. [CrossRef]

19. Liu, G.; Mao, S.; Kim, J.H. A mature-tomato detection algorithm using machine learning and color analysis. Sensors 2019, 19, 2023. [CrossRef]

20. Rahnemoonfar, M.; Sheppard, C. Deep Count: Fruit counting based on deep simulated learning. Sensors 2017, 17, 905. [CrossRef]

21. Chen, S.W.; Shivakumar, S.S.; Dcunha, S.; Das, J.; Okon, E.; Qu, C.; Taylor, C.J.; Kumar, V. Counting apples and oranges with deep learning: A data-driven approach. IEEE Robot. Autom. Lett. 2017, 2, 781-788. [CrossRef]

22. Pan, S.J.; Yang, Q. A survey on transfer learning. IEEE Trans. Knowl. Data Eng. 2010, 22, 1345-1359. [CrossRef] 
23. Bargoti, S.; Underwood, J. Deep fruit detection in orchards. In Proceedings of the 2017 IEEE International Conference on Robotics and Automation (ICRA), Singapore, 29 May-3 June 2017; pp. 3626-3633.

24. He, K.; Zhang, X.; Ren, S.; Sun, J. Deep residual learning for image recognition. arXiv 2015, arXiv:1512.03385.

25. Szegedy, C.; Ioffe, S.; Vanhoucke, V.; Alemi, A. Inception-v4, Inception-ResNet and the Impact of Residual Connections on Learning. arXiv 2016, arXiv:1602.07261.

26. Ren, S.; He, K.; Girshick, R.; Sun, J. Faster R-CNN: Towards real-time object detection with region proposal networks. arXiv 2015, arXiv:1506.01497. [CrossRef] [PubMed]

27. Wang, Z.; Walsh, K.; Verma, B. On-Tree Mango Fruit Size Estimation Using RGB-D Images. Sensors 2017, 17, 2738. [CrossRef] [PubMed]

28. Schillaci, G.; Pennisi, A.; Franco, F.; Longo, D. Detecting Tomato Crops in Greenhouses Using a Vision Based Method. In Proceedings of the International Conference RAGUSA SHWA 2012 on "Safety Health and Welfare in Agriculture and in Agro-food Systems", Ragusa, Italy, 3-6 September 2012; pp. 252-258.

29. Sun, J.; He, X.; Ge, X.; Wu, X.; Shen, J.; Song, Y. Detection of Key Organs in Tomato Based on Deep Migration Learning in a Complex Background. Agriculture 2018, 8, 196. [CrossRef]

30. Liu, G.; Nouaze, J.C.; Touko Mbouembe, P.L.; Kim, J.H. YOLO-Tomato: A Robust Algorithm for Tomato Detection Based on YOLOv3. Sensors 2020, 20, 2145. [CrossRef]

31. Krizhevsky, A.; Sutskever, I.; Hinton, G.E. ImageNet classification with deep convolutional neural networks. Commun. Acm. 2017, 60, 84-90. [CrossRef]

32. Koirala, A.; Walsh, K.B.; Wang, Z.; McCarthy, C. Deep learning for real-time fruit detection and orchard fruit load estimation: Benchmarking of 'MangoYOLO'. Precis. Agric. 2019, 20, 1107-1135. [CrossRef]

33. Ghosal, S.; Zheng, B.; Chapman, S.C.; Potgieter, A.B.; Jordan, D.R.; Wang, X.; Singh, A.K.; Singh, A.; Hirafuji, M.; Ninomiya, S.; et al. A weakly supervised deep learning framework for sorghum head detection and counting. Plant Phenomics 2019, 2019, 1-14. [CrossRef]

34. Desai, S.V.; Chandra, A.L.; Guo, W.; Ninomiya, S.; Balasubramanian, V.N. An adaptive supervision framework for active learning in object detection. arXiv 2019, arXiv:1908.02454.

35. Chandra, A.L.; Desai, S.V.; Balasubramanian, V.N.; Ninomiya, S.; Guo, W. Active Learning with weak supervision for cost-effective panicle detection in cereal crops. arXiv 2019, arXiv:1910.01789.

36. Sørensen, R.A.; Rasmussen, J.; Nielsen, J.; Jørgensen, R. Thistle Detection Using Convolutional Neural Networks. In Proceedings of the 2017 EFITA WCCA Congress, Montpellier, France, 2-6 July 2017; pp. 161-162.

37. Jiang, Z.; Liu, C.; Hendricks, N.P.; Ganapathysubramanian, B.; Hayes, D.J.; Sarkar, S. Predicting County Level Corn Yields Using Deep Long Short Term Memory Models. arXiv 2018, arXiv:1805.12044.

38. You, J.; Li, X.; Low, M.; Lobell, D.; Ermon, S. Deep Gaussian Process for Crop Yield Prediction Based on Remote Sensing Data. In Proceedings of the Thirty-First AAAI Conference on Artificial Intelligence (AAAI-17), San Francisco, CA, USA, 4-9 February 2017; pp. 4559-4565.

39. Shadrin, D.; Pukalchik, M.; Uryasheva, A.; Tsykunov, E.; Yashin, G.; Rodichenko, N.; Tsetserukou, D. Hyper-spectral NIR and MIR data and optimal wavebands for detection of apple tree diseases. arXiv 2020, arXiv:2004.02325.

(C) 2020 by the authors. Licensee MDPI, Basel, Switzerland. This article is an open access article distributed under the terms and conditions of the Creative Commons Attribution (CC BY) license (http://creativecommons.org/licenses/by/4.0/). 